\title{
PROGNOSIS IN INFANTILE IDIOPATHIC SCOLIOSIS
}

\author{
S. KEITH THOMPSON, GEORGE BENTLEY \\ From The Department of Orthopaedic and Accident Surgery, \\ University of Liverpool, and Royal Liverpool Children's Hospital
}

\begin{abstract}
A review was performed of 86 cases of infantile idiopathic scoliosis treated between 1962 and 1979. The single primary curves were classified as resolving, stable, progressive with a low rib-vertebra angle difference (RVAD) and progressive with a high RVAD. Two single primary curves subsequently developed a second curve and 17 were double when first diagnosed. Prognosis was difficult to establish before the age of five years. Only 18 per cent of curves showing progression beyond 50 degrees reached that point before the age of four. Conversely, if a scoliosis of $\mathbf{5 0}$ degrees or more was present before the age of four it always progressed. A more favourable outcome was indicated by male sex, a left-sided curve, a low initial curve measurement, an RVAD of less than 20 degrees in the initial radiograph, and the onset of scoliosis in the first year of life.
\end{abstract}

It has been recognised for many years that idiopathic scoliosis arising in the first three years of life may sometimes resolve (Harrenstein 1930). However, in other cases, the disease may progress leading to severe deformity and cardiorespiratory deficiency (Scott and Morgan 1955). A comprehensive study of 100 patients by Lloyd-Roberts and Pilcher (1965) revealed that 92 per cent of idiopathic scolioses arising in the first year of life resolved spontaneously.

The ability to distinguish between resolving and progressive curves is of great importance. James, Lloyd-Roberts and Pilcher (1959) were unable to differentiate between the two groups except by prolonged observation. They suggested that curves greater than 35 degrees and those that developed compensatory curves above and below the primary one tended to progress. The latter observation was confirmed by Ferreira and James (1972), who also stated that a curve starting in the second and third year of life was inclined to progress. Mehta (1972) made an important observation which she described as the rib-vertebra angle difference (RVAD) (Fig. 1) in a radiological study of 138 children with infantile idiopathic scoliosis. In 80 per cent of resolving curves the RVAD was under 20 degrees and three months after the first radiograph the RVAD was reduced even if the curve showed an increase. In 80 per cent of progressive curves the RVAD was greater than 20 degrees and three months later it was either the same or greater. If the rib head opposite the apical vertebra on the convex side became obscured on the radiograph by vertebral rotation it was said to have moved from Phase 1 to Phase 2 and was shown by Mehta to indicate a progressive curve.

Mehta (1972) further divided progressive infantile idiopathic scoliosis into benign and malignant types. The benign curves were usually in sturdy children while malignant curves were usually in thin children (Mehta 1977). She also stated that resolving curves might either correct early, before the age of two years, or later at seven or eight years of age.

Ferreira and James (1972) confirmed Mehta's findings in a study of 132 cases of infantile idiopathic scoliosis using supine radiographs but experienced some difficulty in measuring the RVAD.

In an attempt to clarify the behaviour of infantile idiopathic scoliosis and the factors involved in progression or remission, a study was made of children attending the scoliosis clinic at the Royal Liverpool Children's Hospital and the Royal Southern Hospital, Liverpool, between 1962 and 1979.

\section{MATERIAL AND METHODS}

Eighty-six children at present attending the clinic have been studied. All had developed an idiopathic scoliosis in the first three years of life. Of the single primary curves 52 were thoracic and 15 were

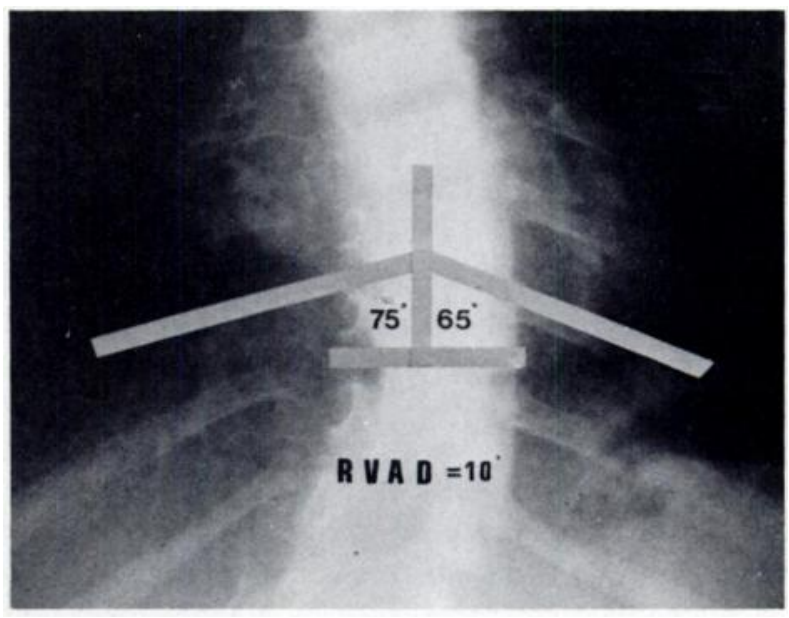

Fig. 1

Measurement of the rib-vertebra angle difference (RVAD).
S. K. Thompson, MChOrth, FRCS Professor G. Bentley, ChM, FRCS
University Department of Orthopaedic and Accident Surgery,

Royal Liverpool Hospital, Prescot Street, Liverpool L7 8XP, England. 
Table I. Analysis of curves in 86 cases of infantile scoliosis

\begin{tabular}{|c|c|c|c|c|c|c|c|c|c|}
\hline Type of curve & $\begin{array}{l}\text { Number } \\
\text { of } \\
\text { children }\end{array}$ & $\mathbf{M}$ & ex & $\begin{array}{l}\text { Sidd } \\
\mathbf{R}\end{array}$ & $\mathbf{L}$ & $\begin{array}{c}\text { Mean age of onset } \\
\text { (months) }\end{array}$ & $\begin{array}{c}\text { Mean initial curve } \\
\text { (degrees) }\end{array}$ & $\begin{array}{l}\text { Mean final curve } \\
\text { (degrees) }\end{array}$ & $\begin{array}{c}\text { RVAD } \\
<\mathbf{2 0} \text { degrees }\end{array}$ \\
\hline \multicolumn{10}{|l|}{ Single curves } \\
\hline Resolving & 24 & 15 & 9 & 5 & 19 & 5.75 & 20 & 2 & 21 (87 per cent) \\
\hline Stable & 19 & 13 & 6 & 8 & 11 & 10.5 & 30 & 29 & 14 (73 per cent) \\
\hline \multicolumn{10}{|l|}{ Progressive } \\
\hline RVAD $<20$ degrees & 8 & 7 & 1 & 2 & 6 & 10 & 31 & 58 & 8 \\
\hline RVAD $>20$ degrees & 16 & 10 & 6 & 7 & 9 & 16 & 51 & 84 & 0 \\
\hline Double curves & 19 & $\varepsilon$ & 11 & $\begin{array}{l}\text { Thora } \\
\text { curv } \\
11\end{array}$ & & 13 & $\begin{array}{cc}\text { Thoracic } & \text { Lumbar } \\
48 & 32\end{array}$ & $\begin{array}{cc}\text { Thoracic } & \text { Lumbar } \\
60 & 47\end{array}$ & - \\
\hline
\end{tabular}

thoracolumbar; none extended above the fourth thoracic vertebra or below the third lumbar vertebra. There were 19 double curves. Children with evidence of cerebral palsy, mental retardation, joint laxity, spina bifida, neurofibromatosis or disorders of connective tissue were excluded.

Early radiographs were taken supine, but when the child was standing and co-operative erect radiographs were obtained. The limits of the curve were determined on the initial radiograph and all subsequent radiographs were measured at these levels. The RVAD was also determined on the initial radiograph. Each child was followed up at regular intervals of three to six months so that a graph of individual progress could be made.

\section{ANALYSIS OF THE CURVES}

\section{Single curves}

It was possible to classify the single primary curves as resolving, stable and progressive. Details of these are given in Table $I$.

Resolving curves (Fig. 2). Of the 24 resolving curves, 23 were diagnosed in the first year of life, and 21 had an RVAD of less than 20 degrees. Minor curves disappeared completely in 11 children and 13 significant curves (20 to 41 degrees) improved to insignificant curves (less than 10 degrees). Only three curves resolved completely before the age of two years. Four curves did not resolve until between 11 and 12 years of age. Nine curves increased by up to 17 degrees before improving. Stable curves (Fig. 3). Nineteen children had curves that were between 10 and 50 degrees which remained stable throughout the period of review. Two children were still under five years of age at review and had curves of 21 and 27 degrees and an RVAD of less than 20 degrees; they may yet prove to have resolving curves. Thirteen curves were diagnosed in the first year of life and 14 had a RVAD of less than 20 degrees.

Treatment of these 19 children varied. Ten were treated conservatively, six by a corrective plaster jacket and Milwaukee brace and four by a Milwaukee brace alone. Nine were treated surgically by a combination of concave release (six), costodesis (nine), convex fusion (two), epiphysiodesis (two) and a Roaf plate (one); after operation they were treated in a plaster jacket for three to six months and then by a Milwaukee brace for variable periods.

Six out of 10 patients treated conservatively and six out of nine treated surgically had no evidence of progression before treatment, demonstrating that the treatment probably did not influence the curve which was intrinsically stable: these curves would therefore be equivalent to Mehta's benign group.

Progressive curves. Twenty-four children had single primary curves progressing beyond 50 degrees, eight of whom had an RVAD of less than 20 degrees.

$R V A D$ less than 20 degrees (Fig. 4). Six of these eight children were diagnosed in the first year of life, and the other two at 1.4 and 1.5 years of age. The initial curve measured between 19 and 45 degrees. The latest follow-up examination was between 10 and 16.8 years of age (average 12.5 years of age) and the curves were then between 50 and 75 degrees. Only one child has not had surgical treatment and he has been treated in a Milwaukee brace. The surgical procedures in the remaining seven children included concave release (three), costodesis (five), epiphysiodesis (two) and Harrington instrumentation with posterior spinal fusion (two). Despite treatment all these patients showed gradual progression of the curvature. Only one child did not have evidence of progression before operation.

In this group an RVAD of less than 20 degrees did not indicate stability but these curves progressed less than those with an RVAD greater than 20 degrees. The great majority of this group were boys, had left-sided curves and had been diagnosed in the first year of life. These factors may be useful in prognosis.

$R V A D$ greater than 20 degrees (Fig. 5). Sixteen children had curves with an RVAD between 26 and 66 degrees on the initial radiograph. Only six of them were diagnosed in the first year of life, the remainder being diagnosed between one and three years of age. The initial curves measured from 21 to 80 degrees. The latest follow-up examination in this group was between the ages of 6.5 and 18.5 years (average 15.2 years) and the curves then ranged from 56 to 115 degrees. 
Fifteen children had had surgical treatment which included concave release (12), costodesis (six), epiphysiodesis (11), a Harrington rod (seven), posterior fusion (eight), convex fusion (five), and a Roaf plate (one). Only three curves had maintained a correction within 10 degrees of the corrected value at the time of review. Despite treatment all showed progression of the curve.

One boy did not have surgical treatment due to parental objection; his curve progressed to 115 degrees despite a plaster jacket, a Parvis brace and a Milwaukee brace.

\section{Double curves}

There were 19 children with double curves (Table I), which were noticed in the first year of life in 10. The upper curve was between the first and twelfth thoracic vertebrae (comprising an average of 6.5 vertebrae) and the lower between the tenth thoracic and the fourth lumbar vertebrae (an average of five vertebrae). Early radiographs were not available in all cases, only nine children having had radiographs taken before the age of five years. Two children had only a single structural curve on the initial radiograph, and in both it was thoracic. The lumbar curves became obvious at two years and nine years of age respectively.

Seventeen children had had surgical treatment which included epiphysiodesis (on two thoracic curves, and five lumbar), posterior fusion (eight), a Roaf plate (eight), concave release (11), costodesis (11), a Harrington rod (two) and an iliolumbar bony strut (one). One child died from a chest infection at the age of 14 years.

It was notable that one lumbar curve resolved after epiphysiodesis but only three other curves improved by 10 to 20 degrees (average 16 degrees). The rest progressed or remained unchanged.

\section{DISCUSSION}

The appearance of an infantile scoliosis causes great concern to parents and to orthopaedic surgeons. Knowledge of the natural history of the idiopathic form of the disease should enable a more rational approach to treatment to be taken (James et al. 1959; Lloyd-Roberts

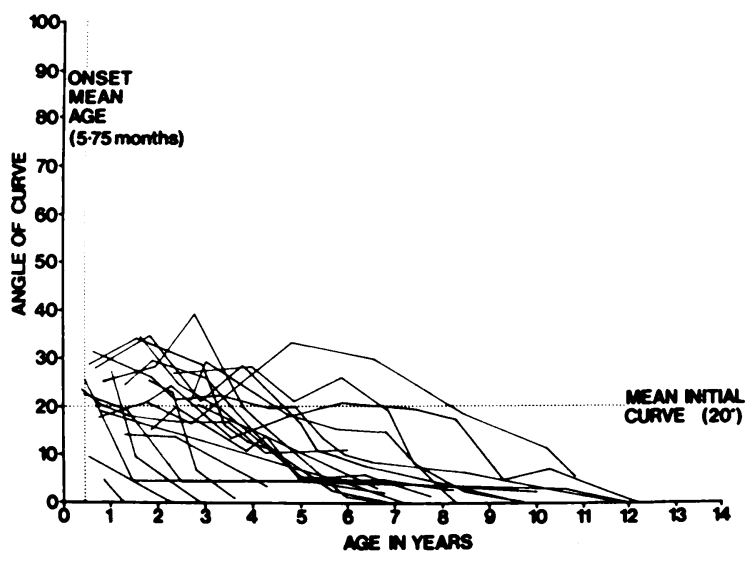

Fig. 2

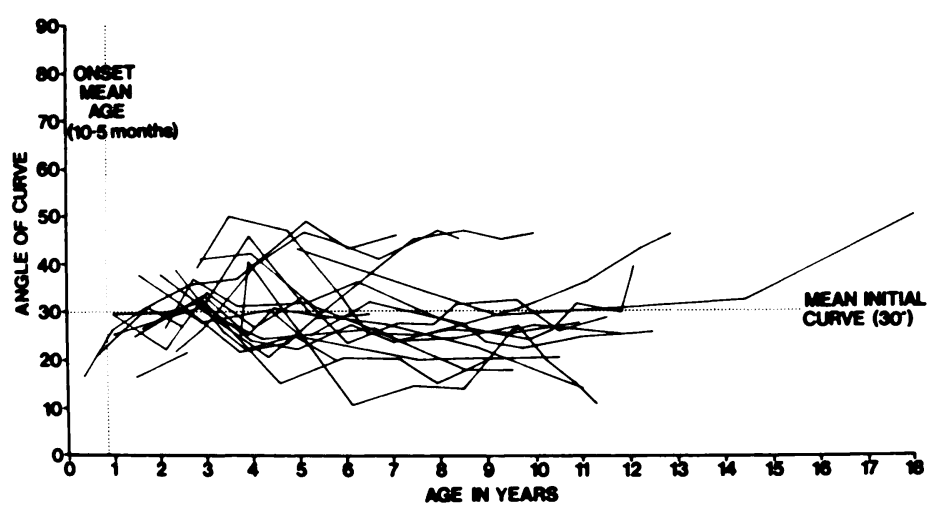

Fig. 3

Figure 2-Progress of resolving infantile idiopathic scoliosis. Figure 3-Progress of stable infantile idiopathic scoliosis.

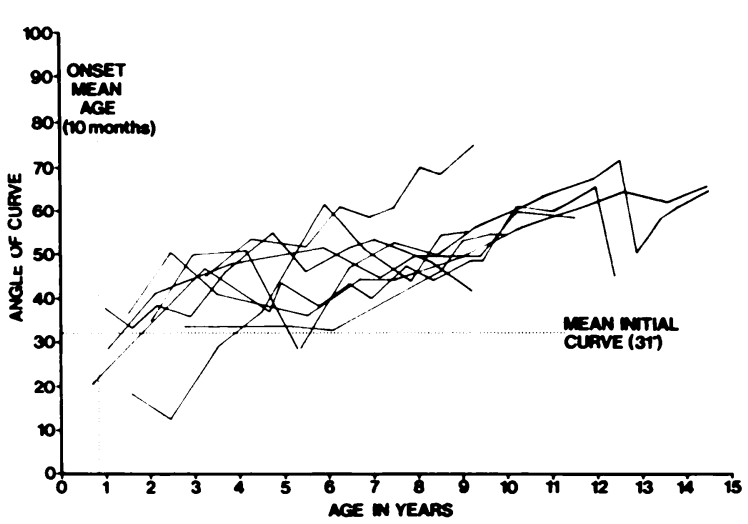

Fig. 4

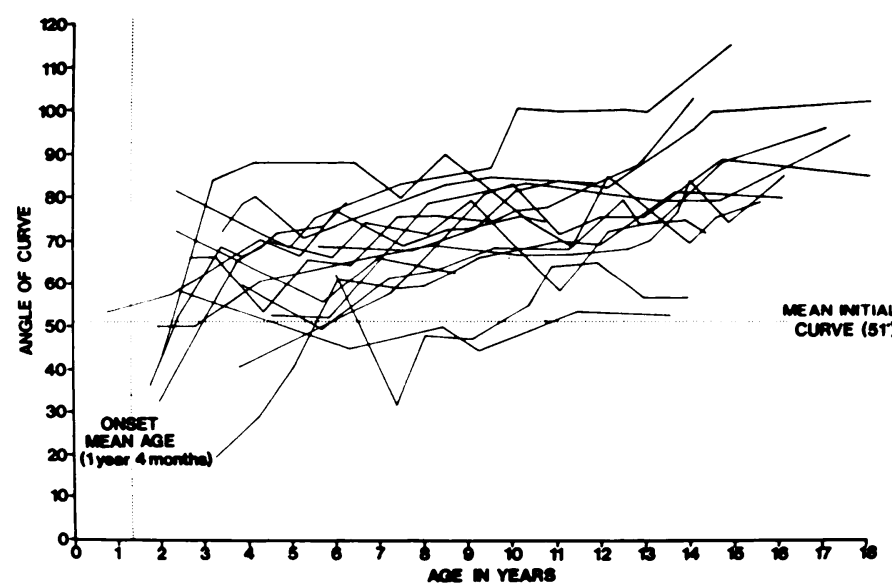

Fig. 5

Figure 4-Progress of progressive infantile idiopathic scoliosis with an RVAD less than 20 degrees. Figure 5-Progress of progressive infantile idiopathic scoliosis with an RVAD greater than 20 degrees. 
and Pilcher 1965; Ferreira and James 1972; Mehta 1972, 1977).

The true incidence of infantile resolving scoliosis is unknown since many minor and resolving curves are probably never diagnosed. As a result the patients in a study such as this are selected.

It is apparent from this study that a structural thoracic or thoracolumbar curve appearing in the first three years of life may undergo several courses (Figs 2 to $5)$. The curve may resolve within the first 12 years of life, especially if the RVAD is less than 20 degrees. The curve may remain "stable", that is between 10 and 50 degrees, even as far as puberty; these curves probably correspond to Mehta's benign progressive curves. The curve may progress beyond 50 degrees and become severe. A second structural curve may appear in the thoracolumbar or lumbar region; this happened in only two children in this series.

Factors in the prognosis of infantile idiopathic scoliosis Sex. Out of 67 single curves 45 were in boys and 22 in girls, a ratio of 2:1. The figures for each group show that the majority of mild curves were in boys. With double curves the sex distribution was slightly reversed, there being eight boys and 11 girls.

Side and severity of curvature. The percentage of left-sided curves in the various groups were: resolving, 79 per cent; stable, 58 per cent; progressive with low RVAD, 75 per cent; and progressive with a high RVAD, 56 per cent. The average initial curve increased in the following group order: resolving (20 degrees), stable (30 degrees), progressive with a low RVAD (31 degrees) and progressive with a high RVAD (51 degrees). There were, however, some curves of only 20 degrees in all groups.

Rib-vertebra angle difference. An RVAD of less than 20 degrees was found in 87 per cent of resolving curves, in 73 per cent of stable curves and in 33 per cent of progressive curves. The progressive curves with this low RVAD did not progress to the same extent as those with a high RVAD, the mean final curves being 58 degrees and 84 degrees respectively. Thus an RVAD less than 20 degrees indicated a more favourable outcome overall, but was not an absolute indicator of the future behaviour of the curve.
The age at diagnosis. The mean age of onset of the resolving curves was 5.75 months, which was significantly younger than in other groups which ranged from 10 to 16 months (Table I). Onset in the first year therefore carried a more favourable prognosis.

\section{Progress of the primary curve}

Resolving curves either resolve quickly, so that the spine is straight within the first year, or more slowly taking as long as 12 years. In six children, however, the resolving nature of the curve became obvious at an intermediate stage, between the ages of three and six and a half years. The stable curves were all less than 55 degrees by the fifth birthday. Of the progressive curves, those with a low RVAD had a similar pattern to the stable group in the first five years so that the two could not be distinguished and the progressive curve did not increase beyond 50 degrees until six or seven years of age. Although the resolving curves may be differentiated from the progressive in most cases by the age of five years, the differentiation of the stable curves from the progressive may not be definite until the age of 10 years. It is only in the progressive group with a high RVAD that the progressive nature of the scoliosis is obvious within the first four years of life. This was so for only 18 per cent of children in this study.

The number of vertebrae in the primary curve and the position of the curve do not appear to be important in prognosis. Study of the development of compensatory curves was not feasible in this series. All primary curves occupied a similar position and included a similar number of vertebrae: the first three groups averaged 6.3 to 6.5 vertebrae, while the progressive curves with a high RVAD averaged 7.5 vertebrae.

Influence of treatment. In this study overall 31 of 43 resolving or stable curves showed no evidence of progression before treatment, suggesting that the treatment may not have influenced the curve and may have been unnecessary; but 20 of 24 progressive curves progressed despite treatment, suggesting that the treatment used did not influence the curve. Treatment should not be undertaken when the curve is compensated and less than 40 degrees unless progression has been demonstrated by serial radiographs.

We wish to thank Professor R. Roaf who had treated many of the patients, Miss Jean Beddow for her considerable help with collection of the records, Mr K. Biggs and Mr A. Taunton who prepared the illustrations and Miss Susan Kermode who typed the manuscript.

\section{REFERENCES}

Ferreira JH, James JIP. Progressive and resolving infantile idiopathic scoliosis: the differential diagnosis. J Bone Joint Surg [Br] 1972;54-B:648-55.

Harrenstein RJ. Die Skoliose bei, Säuglingen und ihre Behandlung. Z Orthop Chir 1930;52:1.

James JIP, Lloyd-Roberts GC, Pilcher MF. Infantile structural scoliosis. J Bone Joint Surg [Br] 1959;41-B:719-35.

Lloyd-Roberts GC, Pilcher MF. Structural idiopathic scoliosis in infancy. J Bone Joint Surg [Br] 1965;47-B:520-23.

Mehta MH. The rib-vertebra angle in the early diagnosis between resolving and progressive infantile scoliosis. $J$ Bone Joint Surg [Br] 1972;54-B:230-43.

Mehta MH. The natural history of infantile idiopathic scoliosis. In: Zorab PA, ed. Scoliosis. Proceedings of a Fifth Symposium held at the Brompton Hospital, September 1976. London, New York, San Francisco: Academic Press, 1977:103-22.

Scott JC, Morgan TH. The natural history and prognosis of infantile idiopathic scoliosis. J Bone Joint Surg [Br] 1955;37-B:400-13. 